\title{
Research Paper: The Influence of Horizontal Veloc- ity on Inter-lower-limbs Local and Global Asymmetry During Walking
}

\author{
Alireza Nasirzadeh ${ }^{1 *}(1)$
}

1. Department of Sports Biomechnics, Faculty of Sport Sciences and Physical Education, Kharazmi University, Tehran, Iran.

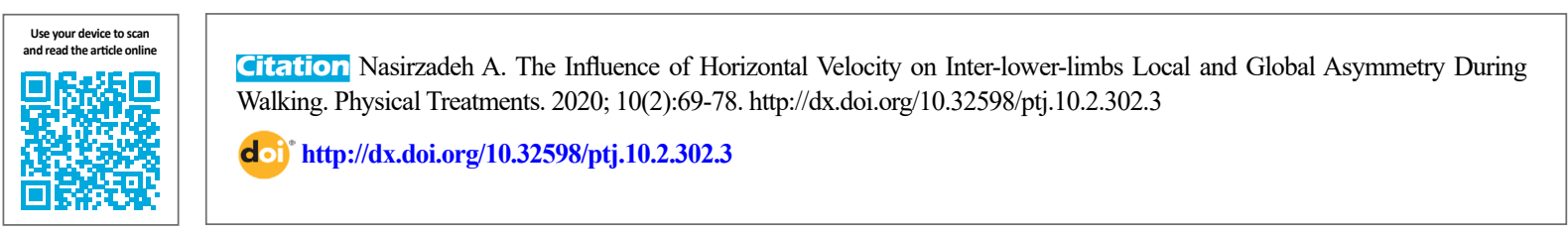

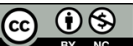

Article info:

Received: 01 Oct 2019

Accepted: 18 Mar 2020

Available Online: 01 Apr 2020

Keywords:

Walking, Gait asymmetry, Movement speed, Principal component analysis

\begin{abstract}
A B S T R A C T
Purpose: Considering the influence of horizontal velocity on many biomechanical characteristics of walking, the purpose of this study was to investigate how inter-lower-limbs local and global asymmetry is influenced by changes in walking speed from slow to fast.

Methods: Ground reaction force data and trajectory of attached markers of bilateral lower limbs of 15 right leg-dominant able-bodied males were collected at each of three walking velocity conditions (slow, normal, and fast). Walking step frequencies were controlled with a metronome. Principal Component Analysis (PCA) was performed on net sagittal joint moments of the stance phase to identify the actions of each joint and the lower limbs separately. Lower limb behavior was assumed symmetrical if the PCA curves extracted from each joint (local gait asymmetry) or each of the lower limbs (global gait asymmetry) described the same portion of the stance phase.
\end{abstract}

Results: Based on findings, the PCA method highlighted different functional tasks for ankle, knee, and hip joints suggesting local gait asymmetry at slow, normal, and fast walking. Also, at slow walking speed, total lower limbs showed global gait asymmetry, however, for normal and fast walking speeds, results showed global gait symmetry.

Conclusion: Considering the possibility of the effect of movement velocity on walking behavior, it is recommended that this factor should be controlled during walking investigations in clinical and research settings.

\footnotetext{
* Corresponding Author: Alireza Nasirzadeh, PhD.

Address: Department of Sports Biomechanics, Faculty of Physical Education and Sport Sciences, Kharazmi University, Tehran, Iran. Phone: +98 (935) 9515557

E-mail: alireza.nasirzade@gmail.com
} 


\section{Highlights}

- Suggestion of local gait asymmetry for lower limbs bilateral joints at slow, normal, and fast walking

- Suggestion of global gait symmetry by increasing the walking speed

- Movement velocity may affect walking behavior

\section{Plain Language Summary}

Movement velocity is an important factor in clinical gait biomechanics, which could be effective on asymmetrical behavior of lower limbs joint and total lower limbs. The previous studies have not provided a thorough commentary on this topic, so in the present study we consider this issue. Our general findings indicate that lower limbs behavior changes when walking at various velocities. Based on this finding we recommended that walking velocity should be considered as a controlling factor in this investigation.

\section{Introduction}

n general, research on walking asymmetry is important as lower limbs should be coordinate to produce a smooth rhythmical walking motion. Gait evaluation can be affected by assuming lower limb symmetry since asymmetry is frequently considered to indicate gait pathology. Therefore, accepting that gait is asymmetrical in the able-bodied population is an important issue for clinicians and may also be an important consideration in physical activities, gait evaluation, clinical decisions for patients requiring rehabilitation for one or both lower extremities, and artificial limb design [1].

There are various methods for the evaluation of gait asymmetry. One of these methods is using Principal Component Analysis (PCA) over different lower limbs waveform data, such as net muscle moment [2] and mechanical power [3] curves. Mathematically, PCA is an orthogonal transformation that converts several correlated variables into a smaller number of uncorrelated, independent variables called principal components [4]. PCA is ideally suited to data reduction, which has the capability of detecting and classifying the main structure of the curve data throughout the variation in the data [5].

Using this method on stance phase muscle moment curves data to identify and compare different actions of bilateral lower limb joints and total lower limbs during able-bodied normal walking, Sadeghi [2] identified different actions at bilateral joints level (local asymmetry) and similar total lower limbs level (global symmetry).
Lower limb symmetry was assumed to be present if the Principal Component (PC) curves derived from each joint or each of the lower limbs described the same portion of the stance phase [2].

From the biomechanical point of view, changing in horizontal velocity is one of the factors that could influence different characteristics of human walking, including bilateral inter-joint and inter-limb asymmetry [6-8]. Presently, no study has been conducted examining the influence of horizontal velocity on the asymmetry of walking from the local and global point of view. Accordingly, the purpose of this study was to determine the influence of horizontal velocity on inter-lowerlimbs local and global asymmetry during walking.

\section{Materials and Methods}

\section{Participants}

Fifteen right-leg-dominant able-bodied males (Mean \pm SD of age: $27.14 \pm 3.67$ years, of weight: $69.84 \pm 6.13 \mathrm{~kg}$, of height: $176.57 \pm 5.06 \mathrm{~cm}, \mathrm{BMI}$ : $22.14 \pm 1.61 \mathrm{~kg} / \mathrm{m}^{2}$ ) volunteered for the present study. Functional leg dominance was determined through kicking a ball and hopping on one leg during a hop test [1]. All participants were thoroughly informed about both the purpose and the process of the study. None of the participants had any reported orthopedic, neurological, or mental disturbances, such as a recent injury or surgery affecting their walking ability. 


\section{Experimental protocol}

Participants were asked to walk barefoot along a 10 $\mathrm{m}$ walkway at 3 different step frequencies that were controlled with a metronome. Step frequencies were set at $108 \mathrm{bpm}$ (average speed; selected similar to ablebodied preferred walking cadence [9], 92 bpm (slow speed; $15 \%$ slower than average speed), and $130 \mathrm{bpm}$ (fast speed; $20 \%$ faster than average speed). Before the data collection, participants were instructed to carry out a series of practice trials with selected walking step frequencies to adjust to the constraints of the walking tasks. After enough practice, each participant performed each walking condition five times and the best three trials were selected based on the following criteria: 1 . the right and left feet made entire contact with the first and second force plates, respectively; 2 . the subject did not noticeably target the force plates; and 3. the subject walked within $\pm 2.5 \%$ of the selected step frequencies [10].

\section{Data collection}

Bilateral gait data were collected using a 6-camera Vicon MX system (Vicon, Oxford, UK), operating at $200 \mathrm{~Hz}$ synchronized to two Kistler force plates (Kistler Instrument AG, Winterthur, Switzerland), sampled at $1000 \mathrm{~Hz}$. The Vicon Plug-in-Gait marker set (PiGVicon Motion Systems, Oxford, UK) was used to generate the kinematic and kinetic data.

\section{Data processing}

A series of fourth-order low-pass zero-phase lag Butterworth filter with the cut-off frequencies of $6 \mathrm{~Hz}$ and $40 \mathrm{~Hz}$ were applied to reduce the noisy marker coordinates and force plate data, respectively [11]. Net sagittal joint moments were calculated at the hip, knee, and ankle of the lower limbs during the stance phase of two consecutive strides of the right and left lower limbs using Visual3D software (CMotion Inc., Rockville, MD). Lower-limb joint moments were normalized concerning body mass and duration of the Gait Cycle (GC) of each lower limb. Joint moments were calculated according to the convention proposed by the International Society of Biomechanics that were extensor at the hip and knee, and plantar flexor at the ankle was assigned a positive direction [11].

\section{Data reduction and statistical analysis}

PCA method was applied to identify the main structure of the data throughout the variation in the data. As described previously [2], PCA was performed twice on net sagittal joint moments of stance phase: once to identify the actions of each joint separately, and a second time to determine symmetry between the lower limbs by simultaneously analyzing all the joints of each limb. Lower limb behavior was assumed symmetrical if the PC curves derived from each joint or each of the lower limbs described the same portion of the stance phase.

Briefly, PCA consists of several steps: Creating three matrices from net joint moment curve data of joints or total lower limbs that consisted of 45 rows, each row representing a single trial of a subject ( 15 subjects $\times 3$ trials) and 65, 61 and 59 Columns (for slow, normal, and fast walking, respectively), which contained the instantaneous muscle moment of that joint during the stance phase calculated at each percent of the GC.

For the simultaneous analysis of all the joints of a lower limb during walking tasks, another three matrices were formed that consisted of 135 rows and 65, 61 and 59 Columns (for slow, normal, and fast walking, respectively). The first 45 rows contained the ankle data of each subject ( 15 subjects $\times 3$ trials), while the remaining 90 rows contained the knee and hip data of the same limb. The 65,61 , and 59 columns represent the instantaneous moments for the stance phase calculated at each percent of the GC.

The Varimax rotation was selected for the PCA method to maximize the variation leading to more interpretable physiological information [12]. After extracting PCs, each PC contained values $(65,61$, and 59 for slow, normal, and fast walking, respectively) that have a factor loading. By plotting against the stance phase duration, the PC curves were obtained, as shown in Figures 1-3. In the present study, the first two PCs, which accounted for over $80 \%$ of the variance, were considered for further analysis. The other extracted PCs, which accounted for the remaining variations were not regarded because they presented random variations [13], which are difficult to interpret.

For these two first PCs, a factor loading of higher than 0.70 was used for further biomechanical interpretation [14]. Gait symmetry between two corresponding lower limb joints (local gait symmetry) could be quantified using the PC curves if the significant factor loading (0.7 or over) was similarly distributed in the stance phase. Symmetry of lower limbs (global gait symmetry) is thought to be present if each of the two corresponding representative curves (PCs) derived from the right and 
left limbs describe the same portion of the stance phase with a loading factor of 0.7 or over [15].

Results are expressed as Mean \pm SD. Normal distribution of data was checked with the KolmogorovSmirnov test and normal distribution was found in all spatiotemporal parameters subgroups. Paired-samples t-test was used for comparison between the right and left limb spatiotemporal parameters. Statistical significance was set at $\mathrm{P}<0.05$.

\section{Results}

Table 1 presents primary spatiotemporal measures, including step frequency, speed, stride length, and stance time for the right and left limbs separately across all slow, normal, and fast walking speeds for the healthy participants. There were no significant differences between the right and left limb spatiotemporal parameters through different levels of walking speed.

The eigenvalues related to the variance of the muscle moment data extracted by each PC and its corresponding GC are presented in Table 2. Based on the information presented in Table 2 and Figure 1, in the slow walking speed, the first and second PCs did not describe the same range of GCs in any of the joints of the ankles, knees, and hips (local gait asymmetry). Also, regarding the whole muscle moment curve calculated for the right and left lower limbs, although PC1 highlighted the same range of $\mathrm{GC}, \mathrm{PC} 2$ accounted for different parts of the GC (global gait asymmetry).

At normal walking speed (Figure 2), PC1 and PC2 did not describe the same portion of GCs in any of the joints of the ankles, knees, and hips (local gait asymmetry). For the whole lower limbs, the significant factor load- ing $(0.7$ or over) was distributed between almost the same ranges of the $\mathrm{GC}$ in both the first corresponding PCs (global gait symmetry). There were similar conditions for the fast walking speed (Figure 3), as local gait asymmetry and global gait symmetry was observed.

\section{Discussion}

The purpose of the present study was to investigate the influence of horizontal velocity on inter-lowerlimbs local and global asymmetry during walking. The results demonstrated no significant differences between the right and left lower limbs spatiotemporal parameters at slow, normal, and fast walking speeds. We considered gait symmetry if the first and second extracted PCs of the right and left values highlighted the same range of the GC. Based on this assumption, different portions of GC were highlighted for lower limb joints at slow, normal, and fast walking speeds, suggesting local gait asymmetry. Regarding the whole lower limbs, at slow walking speed, global gait asymmetry was identified; however, for normal and fast walking speeds, the results showed global gait symmetry.

There are various ways to examine the symmetry of lower limbs during walking, of which the most common way is comparing the single parameter, such as peak points of kinematic and kinetic curves between lower limb joints [1]. Another method for the assessment of symmetry of lower limbs is to investigate the whole path of the kinematic and kinetic curves using methods, such as PCA to detect the main structure of the waveform data [4]. Many researchers in biomechanical studies of walking have used this method [2, $3,15]$. Several studies also have used this method to identify the functional tasks of the joints and lower extremities during walking [2, 15-17].

Table 1. Spatiotemporal parameters of the slow, normal, and fast walking

\begin{tabular}{|c|c|c|c|c|c|}
\hline \multicolumn{2}{|c|}{ Variales } & \multicolumn{4}{|c|}{ Mean \pm SD } \\
\hline Speed & Limb Side & Frequency $(\mathrm{Hz})$ & Speed $(\mathrm{m} / \mathrm{s})$ & Stride Length (m) & Stance Phase (\%) \\
\hline \multirow{3}{*}{ Slow } & Right & $93.58 \pm 2.46$ & $1.06 \pm 0.06$ & $1.27 \pm 0.06$ & $65.31 \pm 1.33$ \\
\hline & & & & & \\
\hline & Left & $93.43 \pm 2.75$ & $1.10 \pm 0.09$ & $1.30 \pm 0.05$ & $64.92 \pm 1.48$ \\
\hline \multirow{2}{*}{ Normal } & Right & $109.22 \pm 2.79$ & $1.32 \pm 0.15$ & $1.33 \pm 0.07$ & $61.35 \pm 1.71$ \\
\hline & Left & $109.48 \pm 2.48$ & $1.34 \pm 0.13$ & $1.35 \pm 0.05$ & $61.12 \pm 1.33$ \\
\hline \multirow{3}{*}{ Fast } & Right & $129.44 \pm 3.28$ & $1.65 \pm 0.16$ & $1.44 \pm 0.08$ & $59.13 \pm 1.52$ \\
\hline & & & & & \\
\hline & Left & $129.12 \pm 4.08$ & $1.63 \pm 0.13$ & $1.41 \pm 0.09$ & $59.42 \pm 1.42$ \\
\hline
\end{tabular}


Table 2. The variance extracted by each Principal Component (PC) and its corresponding Gait Cycle (GC)

\begin{tabular}{|c|c|c|c|c|c|c|c|}
\hline \multirow{2}{*}{ Speed } & \multirow{2}{*}{ Joint } & \multicolumn{3}{|c|}{ Percent of Right Lower Limb } & \multicolumn{3}{|c|}{ Percent of Left Lower Limb } \\
\hline & & PC1 & PC2 & Total & PC1 & PC2 & Total \\
\hline \multirow{9}{*}{ Slow } & Ankle & 40 & 22 & 62 & 39 & 25 & 64 \\
\hline & Percent of GC & $9-32$ & $51-62$ & - & $6-28$ & $34-48$ & - \\
\hline & Knee & 45 & 17 & 62 & 58 & 22 & 80 \\
\hline & Percent of GC & $14-40$ & $58-65$ & - & $22-59$ & $8-21$ & - \\
\hline & & & & & & & \\
\hline & Hip & 53 & 31 & 84 & 49 & 38 & 87 \\
\hline & Percent of GC & $2-38$ & $39-57$ & - & $1-30$ & $40-59$ & - \\
\hline & Lower limb & 64 & 17 & 81 & 61 & 21 & 82 \\
\hline & Percent of GC & $19-62$ & $8-18$ & - & $21-61$ & $1-9$ & - \\
\hline \multirow{9}{*}{ Normal } & Ankle & 49 & 29 & 78 & 52 & 35 & 87 \\
\hline & Percent of GC & $1-34$ & $36-50$ & - & $3-38$ & $42-61$ & - \\
\hline & Knee & 45 & 23 & 68 & 52 & 19 & 71 \\
\hline & Percent of GC & $15-39$ & $55-62$ & - & $16-56$ & $1-10$ & - \\
\hline & & & & & & & \\
\hline & Hip & 52 & 42 & 94 & 56 & 32 & 88 \\
\hline & Percent of GC & $27-61$ & $2-26$ & - & $21-57$ & $1-20$ & - \\
\hline & Lower limb & 61 & 19 & 80 & 60 & 18 & 78 \\
\hline & Percent of GC & $21-59$ & $8-19$ & - & $21-59$ & $8-18$ & - \\
\hline \multirow{9}{*}{ Fast } & Ankle & 39 & 29 & 68 & 43 & 32 & 75 \\
\hline & Percent of GC & $9-28$ & $45-59$ & - & $8-32$ & $36-54$ & - \\
\hline & Knee & 46 & 39 & 85 & 42 & 35 & 77 \\
\hline & Percent of GC & $6-30$ & $35-59$ & - & $7-26$ & $38-59$ & - \\
\hline & & & & & & & \\
\hline & Hip & 60 & 24 & 84 & 48 & 45 & 93 \\
\hline & Percent of GC & $30-59$ & $3-28$ & - & $17-55$ & $2-15$ & - \\
\hline & Lower limb & 59 & 21 & 80 & 59 & 20 & 79 \\
\hline & Percent of GC & $20-57$ & $8-19$ & - & $20-57$ & $8-19$ & - \\
\hline
\end{tabular}

Based on the available information, only one study applied PCA to detect lower limb joints' functional tasks during normal walking in able-bodied participants to identify the existence of joint and limb symmetry [2]. This study examined the natural walking speed (1.30 $\mathrm{m} / \mathrm{s}$ ), which is very close to the normal walking speed in the present study. Based on the results of this study, although different tasks were identified using the first and second PCs at the ankle, knee, and hip joints, the PC plots extracted the same range of GC for both lower limbs.

As a result, this study indicated local gait asymmetry and global gait symmetry during normal walking in healthy individuals, which is similar to the findings of the present study. However, the range of the GC extracted by PCA for joints and lower limbs differed between the two studies, which can be due to different test con- 

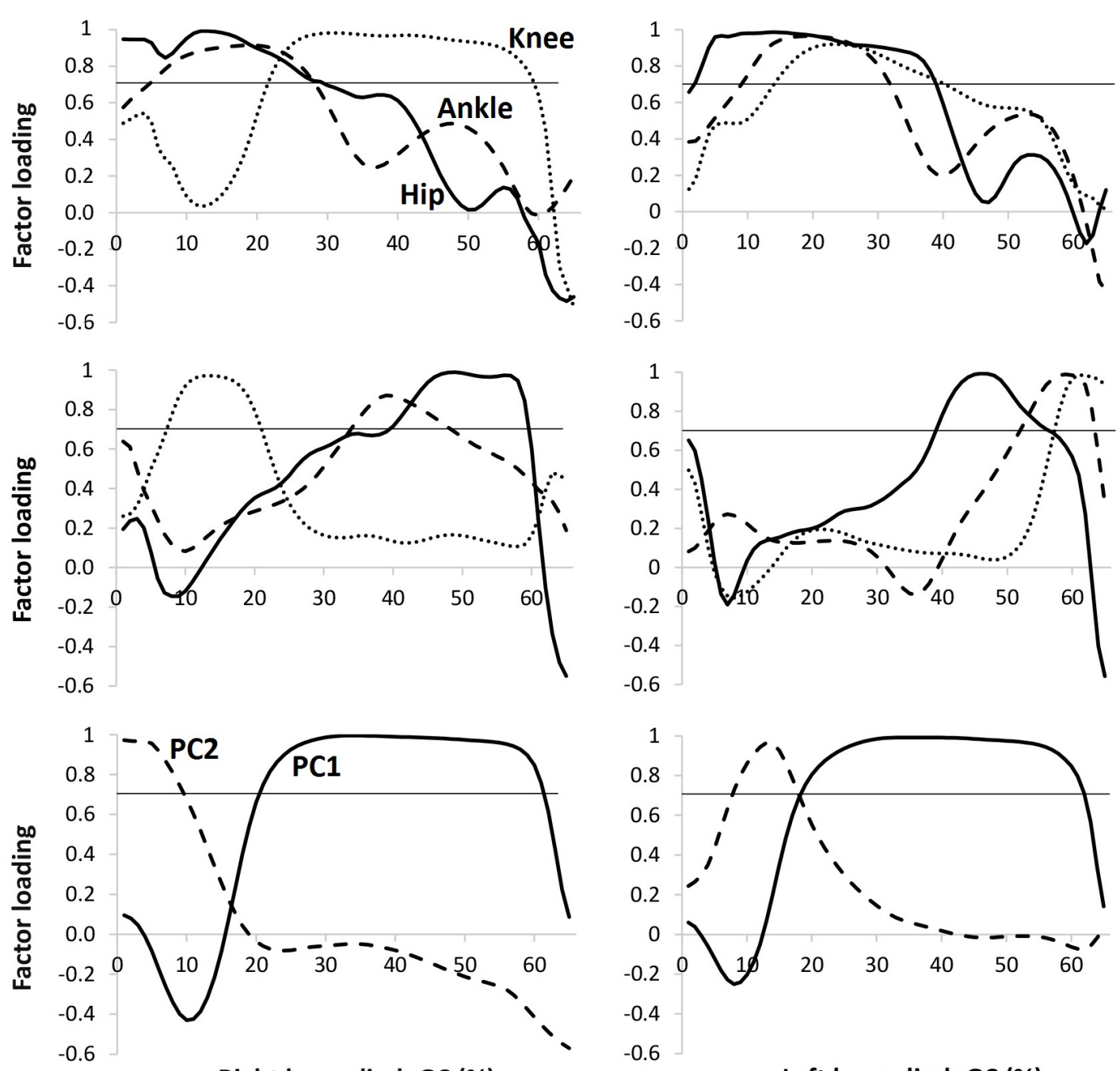

Right lower limb GC (\%)

Left lower limb GC (\%)

PHYSICAL TREA $\|$ MENTS

Figure 1. The first two Principal Components (PCs) extracted from muscle moment curves

Calculated at the right and left ankles, knees, and hips (up and middle) and lower limbs (bottom) during slow walking.

ditions. It should be noted that the action of the lower limbs during walking occurs as a result of the interaction between the ankle, knee, and hip joints, which can be achieved through very different complex patterns [18].

Based on the results of these two studies, it seems that in natural walking speed of able-bodied people, despite the occurrence of different behaviors in lower limb joints that occur as a result of different activities of muscle groups, the overall results of these activities lead to asymmetric behavior of the lower limbs. This condition can explain the findings of studies indicating that the asymmetry of the parameters measured at the level of the lower limb joints (such as peaks of the angular displacement, muscle moment, and power curves), despite the symmetry of the parameters measured from the action of whole lower limbs (such as spatiotemporal parameters) $[2,10,16,19,20]$. The best explanation for the occurrence of global gait symmetry while local gait symmetry, might be the compensatory mechanisms of the central nervous system $[2,16]$. Due to the very complexity of the involvement of these mechanisms, it is still unclear how this action will occur during walking, which requires further research in this area.

One of the important findings of this study was the improvement of walking symmetry with an increase in the speed of motion. There are several examples of enhanced inter-limb coupling with increases in velocity within the domain of dynamical systems theory and motor control [21]. In this domain, it is proposed that the segments of the human body act as coupled oscillators, where the 

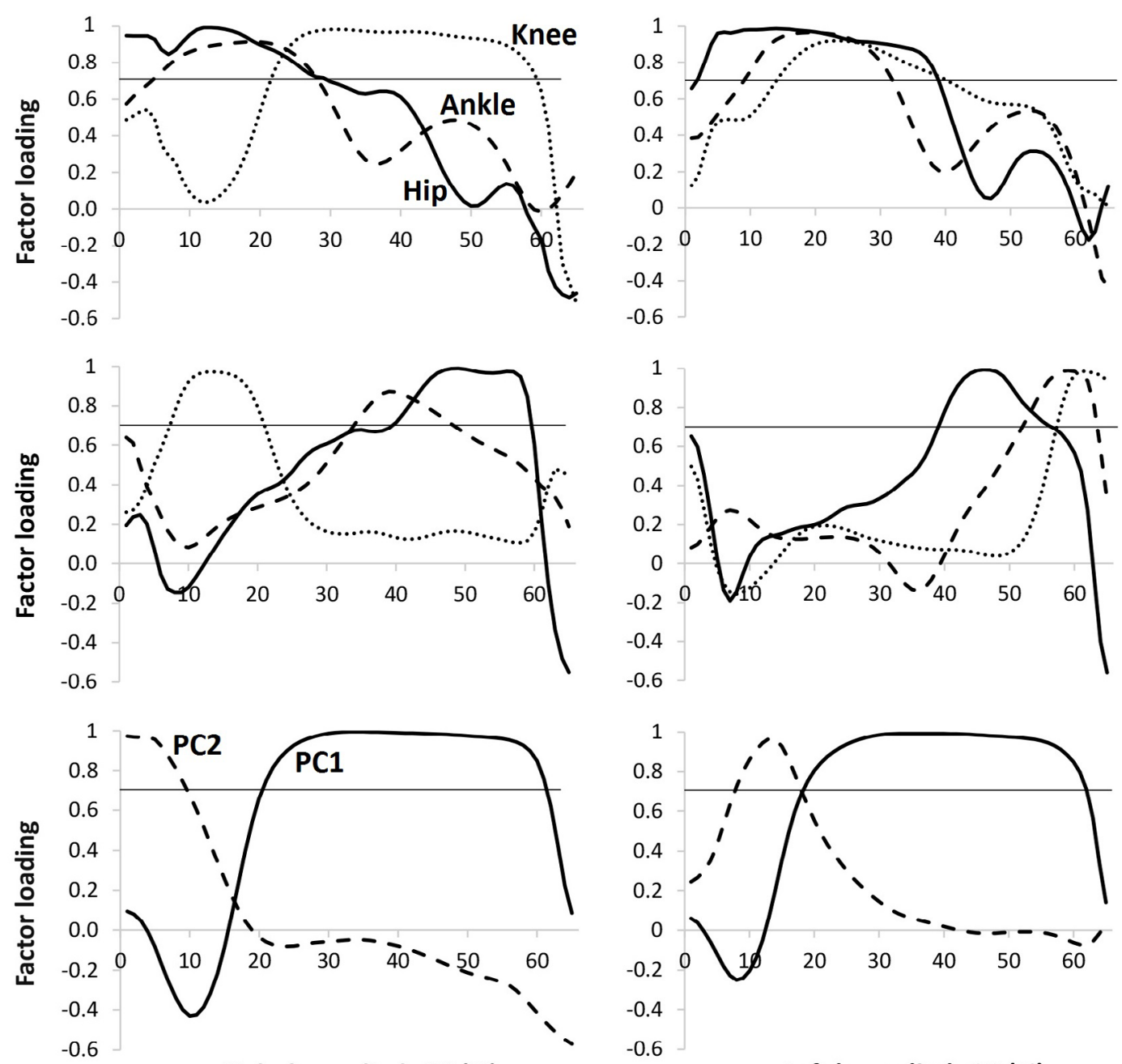

Right lower limb GC (\%)

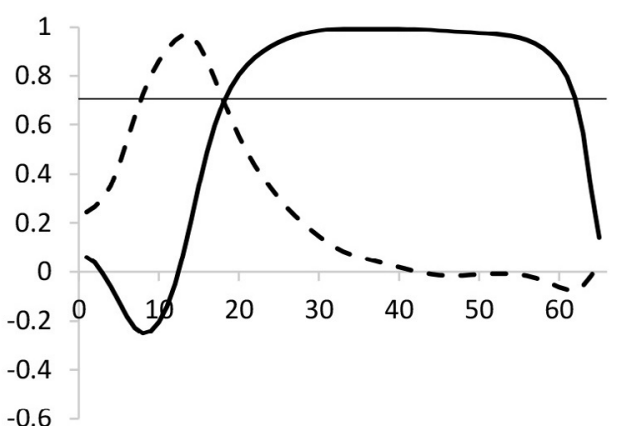

Left lower limb GC (\%)

Figure 2. The first two Principal Components (PCs) extracted from muscle moment curves

PHYSICAL TREATMENTS

Calculated at the right and left ankles, knees, and hips (up and middle) and lower limbs (bottom) during normal walking.

symmetrical behaviors are more easily maintained at higher velocities. At slow speeds, the legs are allowed to decouple and employ different functional strategies, whereas at greater velocities, more highly coupled patterns of movement exists [6]. As a result, it seems that higher walking speeds can lead to the improvement of inter-limb symmetry, while different actions are taken by the lower limbs at lower walking speeds.

Another explanation for this result is the attention and cognitive interference during the slow walking condition. Unlike fast walking, slowing gait is less frequent during daily living. Therefore, slow walking requires more attention that could lead to higher variability that may also contribute to the reduction of bilateral symmetry [8].

The short distance walkway was one of the limitations of this study, which did not allow us to increase the speed of walking more than that. Finally, care should be taken because it is not known if the results obtained from one population can be safely applied to a different population.

\section{Conclusion}

In conclusion, the findings of PCA analysis showed that during normal walking for healthy people, although different actions are taken by the joints (local gait asymmetry), bilateral lower limbs almost exhibit similar behavior (global gait symmetry). Also, with increasing walking speed from slow to fast, despite the lack of changes in local asymmetry of the joints, at the level of total lower limbs, the global symmetry improved. Considering the possibility of the effect of movement velocity, it is recommended that this factor should be controlled during investigating the walking behavior in clinical and research settings. 

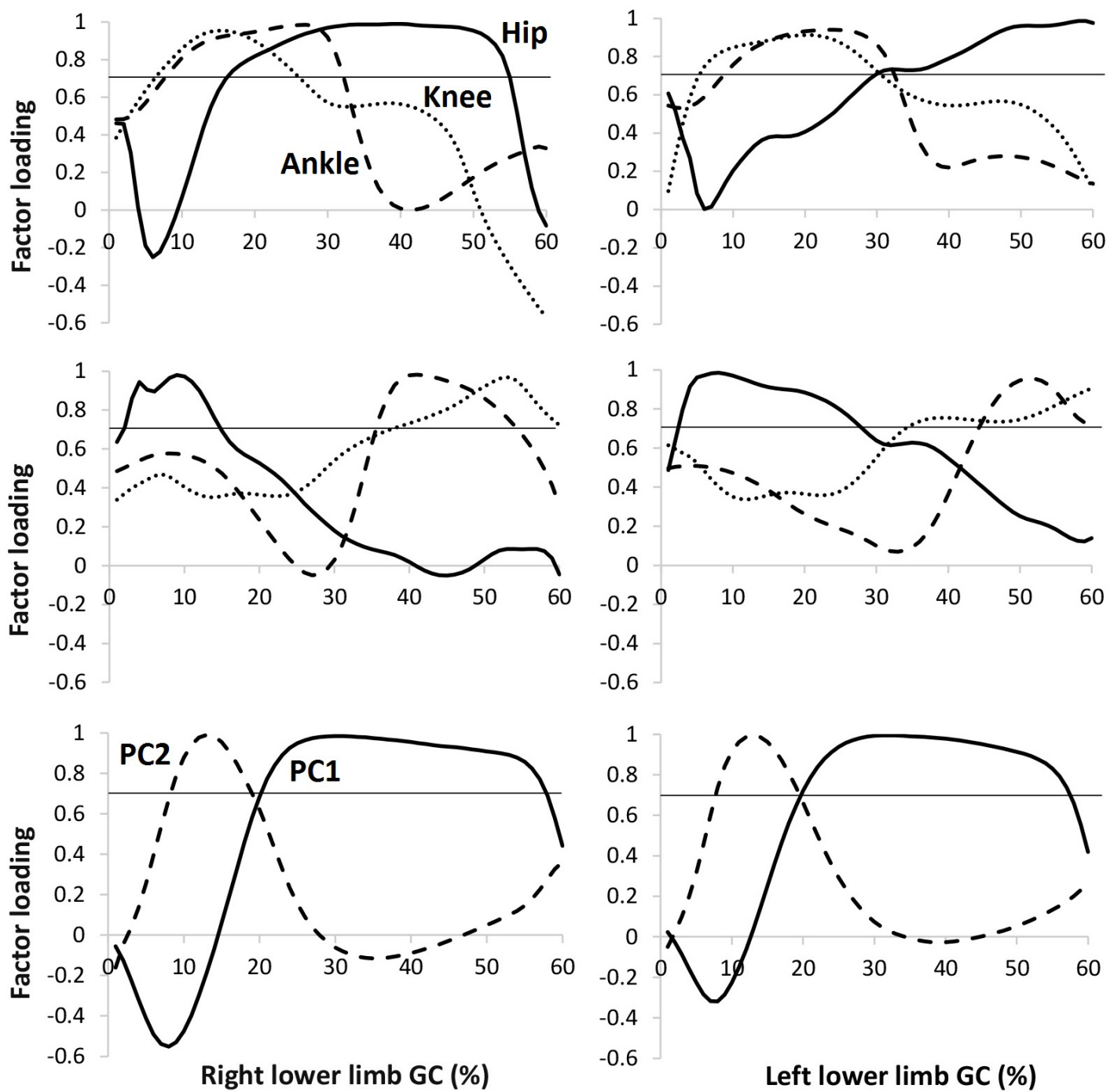

PHYSICAL TREA $\pitchfork$ MENTS

Figure 3. The first two Principal Components (PCs) extracted from muscle moment curves

Calculated at the right and left ankles, knees, and hips (up and middle) and lower limbs (bottom) during fast walking.

\section{Ethical Considerations}

\section{Compliance with ethical guidelines}

The study was approved by the local University Ethics Committee.

\section{Funding}

This research did not receive any grant from funding agencies in the public, commercial, or non-profit sectors.

\section{Conflict of interest}

The author declared no conflict of interest.

\section{Acknowledgments}

The author would like to thank Behnaz Mohebi for her kindly contribution.

\section{Authors' contributions}

All authors contribute in preparing this article. 


\section{References}

[1] Sadeghi H, Allard P, Prince F, Labelle H. Symmetry and limb dominance in able-bodied gait: A review. Gait and Posture. 2000; 12(1):34-45. [DOI:10.1016/S09666362(00)00070-9] [PMID]

[2] Sadeghi H. Local or global asymmetry in gait of people without impairments. Gait and Posture. 2003; 17(3):197204. [DOI:10.1016/S0966-6362(02)00089-9] [PMID]

[3] Sadeghi H. Contributions of lower-limb muscle power in gait of people without impairments. Physical Therapy. 2000; 80(12):1188-96. [DOI:10.1093/ptj/80.12.1188] [PMID]

[4] Deluzio KJ, Harrison AJ, Coffey N, Caldwell GE. Analysis of biomechanical waveform data. In: Robertson DGE, Caldwell GE, Hamill J, Kamen G, Whittlesey SN, editors. Research Methods in Biomechanics. Champaign: Human Kinetics; 2014. [DOI:10.5040/9781492595809.ch-014]

[5] Hamilton LC. Regression with graphics: A second course in applied statistics. Pacific Grove: Duxbury Press; 1992. https:// books.google.com/books?id=6QfvAAAAMAAJ\&q

[6] Goble DJ, Marino GW, Potvin JR. The influence of horizontal velocity on interlimb symmetry in normal walking. Human Movement Science. 2003; 22(3):271-83. [DOI:10.1016/ S0167-9457(03)00047-2]

[7] Patterson KK, Nadkarni NK, Black SE, McIlroy WE. Gait symmetry and velocity differ in their relationship to age. Gait and Posture. 2012; 35(4):590-4. [DOI:10.1016/j.gaitpost.2011.11.030] [PMID] [PMCID]

[8] Plotnik M, Bartsch RP, Zeev A, Giladi N, Hausdorff JM. Effects of walking speed on asymmetry and bilateral coordination of gait. Gait and Posture. 2013; 38(4):864-9. [DOI:10.1016/j.gaitpost.2013.04.011] [PMID] [PMCID]

[9] Cavagna GA, Franzetti P. The determinants of the step frequency in walking in humans. The Journal of Physiology. 1986; 373(1):235-42. [DOI:10.1113/jphysiol.1986.sp016044] [PMID] [PMCID]

[10] Nasirzade A, Sadeghi H, Mokhtarinia HR, Rahimi A [The influence of movement velocity on inter-lower-limbs kinematical symmetry in normal walking: Pilot study (Persian)]. Scientific Journal of Rehabilitation Medicine. 2017; 5(4):159-72. https://www.sid.ir/en/Journal/ViewPaper.aspx?ID=535803

[11] Winter DA. Biomechanics and motor control of human movement. Hoboken: John Wiley \& Sons; 2009. [DOI:10.1002/9780470549148]

[12] Davis BL, Vaughan CL. Phasic behavior of EMG signals during gait: Use of multivariate statistics. Journal of Electromyography and Kinesiology. 1993; 3(1):51-60. [DOI:10.1016/1050-6411(93)90023-P]

[13] Olney SJ, Griffin MP, McBride ID. Multivariate examination of data from gait analysis of persons with stroke. Physical Therapy. 1998; 78(8):814-28. [DOI:10.1093/ $\mathrm{ptj} / 78.8 .814]$ [PMID]

[14] Sadeghi H, Prince F, Zabjek KF, Allard P. Sagittal-hipmuscle power during walking in old and young ablebodied men. Journal of Aging and Physical Activity. 2001; 9(2):172-83. [DOI:10.1123/japa.9.2.172]
[15] Sadeghi H, Prince F, Sadeghi S, Labelle H. Principal component analysis of the power developed in the flexion/ extension muscles of the hip in able-bodied gait. Journal of Medical Engineering and Physics. 2000; 22(10):703-10. [DOI:10.1016/s1350-4533(01)00010-8 ] [PMID]

[16] Sadeghi H, Allard P, Duhaime M. Functional gait asymmetry in able-bodied subjects. Human Movement Science. 1997; 16(2-3):243-58. [DOI:10.1016/S0167-9457(96)00054-1]

[17] Sadeghi H, Sadeghi S, Prince F, Allard P, Labelle H, Vaughan CL. Functional roles of ankle and hip sagittal muscle moments in able-bodied gait. Clinical Biomechanics. 2001; 16(8):688-95. [DOI:10.1016/S0268-0033(01)00058-4]

[18] Winter DA. Overall principle of lower limb support during stance phase of gait. Journal of Biomechanics. 1980; 13(11):923-7. [DOI:10.1016/0021-9290(80)90162-1] [PMID]

[19] Ounpuu S, Winter DA. Bilateral electromyographical analysis of the lower limbs during walking in normal adults. Electroencephalography and Clinical Neurophysiology. 1989; 72(5):429-38. [DOI:10.1016/00134694(89)90048-5] [PMID]

[20] Forczek W, Staszkiewicz R. An evaluation of symmetry in the lower limb joints during the able-bodied gait of women and men. Journal of Human Kinetics. 2012; 35:47-57. [DOI:10.2478/v10078-012-0078-5] [PMID] [PMCID]

[21] Beek PJ, Peper CE, Stegeman DF. Dynamical models of movement coordination. Human Movement Science. 1995; 14(4-5):573-608. [DOI:10.1016/0167-9457(95)00028-5] 
This Page Intentionally Left Blank 